\title{
POD-based reduced-order model of an eddy-current levitation problem
}

\author{
MD Rokibul Hasan and Ruth V. Sabariego
}

KU Leuven, Dept. Electrical Engineering (ESAT), EnergyVille, Belgium

rokib.hasandesat.kuleuven.be, ruth.sabariegodesat.kuleuven.be

\begin{abstract}
Summary. In this paper, a proper-orthogonal-decomposition reduced-order model is applied to an eddy-current problem with movement. A classical magnetodynamic finite element formulation based on the magnetic vector potential is used as reference and as starting point to build the reduced models. The TEAM workshop problem 28 is chosen as a test case.
\end{abstract}

\section{Introduction}

The accurate modelling of electromagnetic devices accounting for eddy current effects, movement, nonlinearities,... is a major concern from early design stages. The finite element (FE) method is widely used and versatile for modelling these phenomena. It maybe extremely expensive in terms of computational time and memory, though. The most popular approaches for handling this issue are those based on the extraction of physical parameters (inductances, flux linkages,...) either from simulations or measurements and the construction of look-up tables covering the operating range of the device [1]. Future simulations are performed by simple interpolation, drastically reducing thus the computational cost.

Reduced order (RO) techniques with a more mathematical approach are a feasible alternative, which are gaining interest in electromagnetism [2]. Few works have addressed electromagnetic problems with movement, crucial to model of e.g. actuators, rotating electrical machines. In [3], a permanent magnet synchronous machine is studied via a POD-RO model of a magnetostatic FE formulation. The rotation is taken into account with the locked step approach, so the mesh and number of degree of freedom remain constant.

In this work, we consider a levitation problem with RO techniques, the Team Workshop problem 28 [4] (see Fig. 1). The time-varying magnetic field generated by the coils induces eddy currents in the conducting plate. The ensuing magnetic force moves the plate, which oscillates around an equilibrium levitating position. The FE mesh changes at every new position/time step. Herein a RO projection operator is developed to properly account for the different meshes with a prescribed accuracy.

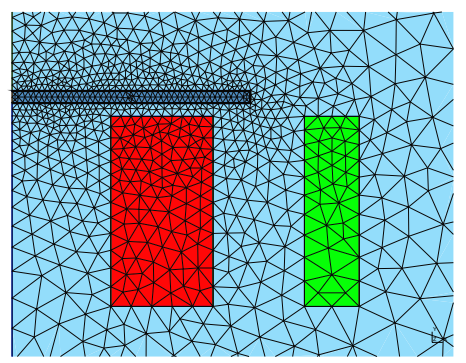

Fig. 1. Team 28 problem: 2D mesh

\section{Magnetodynamic levitation model}

Let us consider a bounded domain $\Omega=\Omega_{c} \cup \Omega_{c}^{C} \in \mathbb{R}^{3}$ with boundary $\Gamma$. The conducting and non-conducting parts of $\Omega$ are denoted by $\Omega_{c}$ and $\Omega_{c}^{C}$, respectively. The (modified) magnetic-vector-potential $(a-)$ magnetodynamic formulation (weak form of Ampère's law) reads: find $a$, such that

$$
\begin{array}{r}
\left(v \operatorname{curl} a, \operatorname{curl} a^{\prime}\right)_{\Omega}+\left(\sigma \partial_{t} a, a^{\prime}\right)_{\Omega_{c}}+\left\langle\hat{n} \times h, a^{\prime}\right\rangle_{\Gamma} \\
=\left(j_{s}, a^{\prime}\right)_{\Omega_{s}}, \forall a^{\prime}
\end{array}
$$

with $a^{\prime}$ test functions in a suitable function space; $b=\operatorname{curl} a$ the magnetic flux density; $j_{s}$ a prescribed current density and $\hat{n}$ the outward unit normal vector on $\Gamma$. Volume integrals in $\Omega$ and surface integrals on $\Gamma$ of the scalar product of their arguments are denoted by $(\cdot, \cdot)_{\Omega}$ and $\langle\cdot, \cdot\rangle_{\Omega}$. The derivative with respect to time is denoted by $\partial_{t}$. We further assume linear isotropic materials with reluctivity $v$ (magnetic field $h=v b$ ) and conductivity $\sigma$.

We adopt a weak electromechanical coupling. Once the electromagnetic problem solved, we compute the magnetic force from $a$ by means of the Maxwell stress tensor. This force is the source of the mechanical problem to determine the plate displacement [5].

$$
m \partial_{t} v(t)+\xi v(t)+k y(t)=F(t), \quad v(t)=\partial_{t} y(t)
$$

where $y(t)$ is the position, $v(t)$ the velocity, $m$ the mass, $\xi$ the viscous friction coefficient, $k$ the elastic constant and $F(t)$ the total applied force including the magnetic forces. 


\section{Model order reduction}

The proper orthogonal decomposition (POD) is applied to reduce the matrix system resulting from the discretisation of [1]:

$$
A \partial_{t} x(t)+B x(t)=C(t) .
$$

The solution vector $x(N \times 1)$ is then approximated by a vector $x^{r}(M \times 1)$ in a reduced basis, $M<N$,

$$
x \approx \Psi x^{r},
$$

with $\Psi$ an orthonormal projection operator generated from the time-domain full solution $x$ via snapshot techniques [6].

Let us consider the snapshot matrix, $S=\left[x_{1}, x_{2}, \ldots\right]$ from the set of solution $x$ for the selected number of time steps. Applying the singular value decomposition (SVD) to this snapshot matrix $S$ as,

$$
\mathscr{S}=\mathscr{U} \Sigma \mathscr{V}^{T}
$$

We consider, $\Psi=\mathscr{U}^{r}$ corresponds to the truncation ( $r$ first columns). Therefore, the reduced-order system becomes:

$$
A^{r} \partial_{t} x^{r}(t)+B^{r} x^{r}(t)=C^{r}(t) .
$$

with $A^{r}=\Psi^{T} A \Psi, B^{r}=\Psi^{T} B \Psi$ and $C^{r}=\Psi^{T} C$ [7]. Note that the FE mesh changes for every snapshot, what means that the projection operator $\Psi$ must be projected to a common basis and the procedure becomes more expensive.

\section{Results}

We consider an electrodynamic levitation device consisting of a conducting plate over two coaxial exciting coils. At $t=0$ the plate rests above the coils at a distance of $3.8 \mathrm{~mm}$. A time-varying sinusoidal current at $50 \mathrm{~Hz}$ is imposed in both coils, same amplitude, opposite directions [4].

We assume a purely translational movement (rotation and tilting are neglected) and adopt a FE axisymmetric model as reference and origin of the RO models.

Reaching steady state has required time-stepping 50 periods (5000 time steps). The first 1400 time-step solutions $(280 \mathrm{~ms})$ are included in the snapshot matrix, as they comprise the first two peaks, see Fig. 2. We have observed that this is the optimal amount of solutions to reproduce the transient.

Three POD-based RO models are constructed with a prescribed error tolerance: $\mathrm{RO} 1, \mathrm{RO} 2, \mathrm{RO} 3$. The smaller the error tolerance, the bigger the RO model will be ( size of $\mathrm{RO} 3<\mathrm{RO} 2<\mathrm{RO} 1$ ).

As preliminary result, the displacement and relative error of the full and RO models are shown in

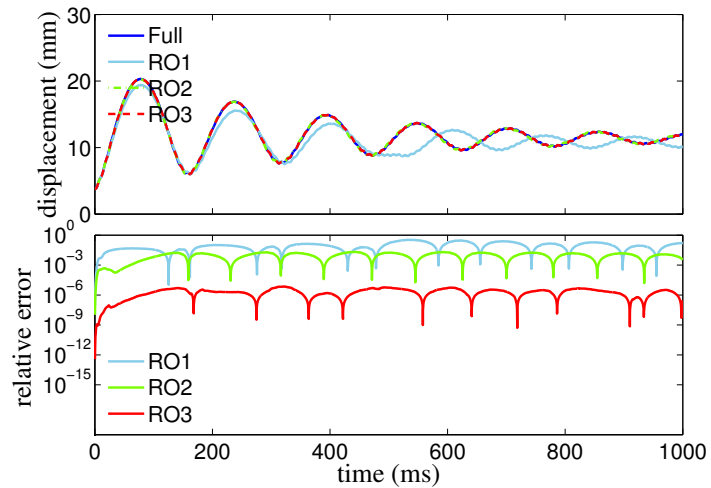

Fig. 2. Displacement (up) and relative error (down) between full and RO models

Fig. 2. With the RO approach we need 1400 time steps instead of the 5000 time steps for getting the full system behaviour. An accurate result has been achieved with the truncated basis models: $\mathrm{RO} 2$ and RO3. Although the RO approach is expensive for a single simulation, the gain in computational cost is clear for repetitive cases.

Details on the theory and a thorough analysis of the behaviour of the RO models with e.g. the time step size and the number of time steps for constructing the projection operator will be provided.

\section{References}

1. Z. Liu, S. Liu, O. A. Mohammed, "A Practical Method for Building the FE-Based Phase Variable Model of Single Phase Transformers for Dynamic Simulations," IEEE Trans. Magn., vol. 43, no. 4, pp. 1761-1764, 2007.

2. W. Schilders, H. V. der Vorst, J. Rommes, "Model order reduction: theory, research aspects and applications," Springer-Verlag, 2008.

3. T. Henneron, S. Clénet, "Model order reduction applied to the numerical study of electrical motor based on POD method taking into account rotation movement," Int. J. Numer. Model., vol. 27, no. 3, pp. 485-494, 2014.

4. H. Karl, J. Fetzer, S. Kurz, G. Lehner, W. M. Rucker, "Description of TEAM workshop problem 28: An electrodynamic levitation device" In Proc. of the TEAM Workshop, Graz, Austria, pp. 48-51, 1997.

5. F. Henrotte, K. Hameyer, "Computation of electromagnetic force densities: Maxwell stress tensor vs. virtual work principle," J. Comput. Appl. Math., vol. 168, pp. 235-243, 2004

6. Y. Sato, H. Igarashi, "Model reduction of threedimensional eddy current problems based on the method of snapshots," IEEE Trans. Magn., vol. 49, no. 5, pp. 1697-1700, 2013.

7. MD R. Hasan, R. V. Sabariego, C. Geuzaine, Y. Paquay, "Proper orthogonal decomposition versus Krylov subspace methods in reduced-order energy-converter models," In Proceedings of ENERGYCON 2016, April 4-8, Leuven, Belgium, 2016. 